\title{
Quantum dot states and optical excitations of edge-modulated graphene nanoribbons
}

\author{
Deborah Prezzi, ${ }^{1, *}$ Daniele Varsano, ${ }^{1, \dagger}$ Alice Ruini, ${ }^{1,2}$ and Elisa Molinari ${ }^{1,2}$ \\ ${ }^{1}$ Centro S3, Istituto Nanoscienze Consiglio Nazionale delle Ricerche, I-41125 Modena, Italy \\ ${ }^{2}$ Dipartimento di Fisica, Università di Modena e Reggio Emilia, I-41125 Modena, Italy
}

(Received 13 May 2011; published 5 July 2011)

\begin{abstract}
We investigate from first principles the electronic and optical properties of edge-modulated armchair graphene nanoribbons, including both quasiparticle corrections and excitonic effects. Exploiting the oscillating behavior of the ribbon energy gap, we show that minimal width-modulations are sufficient to obtain confinement of both electrons and holes, thus forming optically active quantum dots with unique properties, such as the coexistence of dotlike and extended excitations and the fine tunability of optical spectra, with great potential for optoelectronic applications.
\end{abstract}

DOI: 10.1103/PhysRevB.84.041401

PACS number(s): 78.67.Hc, 73.22.-f, 78.40.Ri

Graphene nanostructures have recently triggered a wealth of studies for their remarkable properties, which combine the unique electronic and mechanical features of graphene ${ }^{1}$ with the semiconducting behavior induced by quantum confinement. ${ }^{2}$ Moreover, depending on the details of the atomic structure, a variety of peculiar width and edgerelated phenomena can arise. ${ }^{3}$ To fully exploit this richness, much effort has been devoted to achieve precise control of the structure through a number of different nanofabrication techniques. ${ }^{4}$ Extreme control of the width-down to three benzene rings - was recently demonstrated by chemical routes for armchair-edge graphene nanoribbons (AGNRs), ${ }^{5}$ whose optical gaps ${ }^{6-9}$ finally reach the energy window attractive for optoelectronic applications. The perspective of engineering one-dimensional (1D) and 0D quantum confinement of charge carriers, so far dominated by unintentional disorder effects, ${ }^{10}$ is thus becoming realistic. ${ }^{11}$

Among the different routes proposed to achieve quantum confinement in all directions, ${ }^{12}$ i.e., quantum dots (QDs), the most widely used takes advantage of high-resolution lithography to carve the full device from 2D graphene. ${ }^{13}$ This allows the combination of atomiclike properties, extensively explored in conventional semiconductor QDs, ${ }^{14}$ with the advantages born by graphene, such as efficient coupling to a graphene-based interconnecting wire or contact and planar geometry that is compatible with available technologies. In such a configuration, both the dot and the barriers are made of graphene by modulating the wire width and the carriers end up being localized in the wider region by quantum size effects, as in conventional semiconductor nanostructures.

Here we show that not only quantum size effects, but also a different mechanism, similar to that occurring in conventional semiconductor heterojunctions, can give rise to the confinement of both electrons and holes, where the confining potential landscape is obtained by exploiting the peculiar electronic properties of AGNRs. In fact, the energy band gap of AGNRs shows three distinct families depending on the ribbon width, namely, $N=3 p, N=3 p+1$, and $N=3 p+2$ ( $N$ being the number of dimeric lines along the width and $p$ a positive integer): ${ }^{3}$ Within each family, the energy gap decreases with increasing ribbon width, as expected, but minimal modulations of the width, down to one atomic row, are sufficient to induce a large variation in the band gap. For example, the conduction- and valence-band offsets between the $N=7$ and $N=8$ ribbon are both as large as $1.4 \mathrm{eV}$, as will be discussed below (see Fig. 1).

Joining such ribbons is therefore expected to produce an all-graphene system with type-I band modulation similar to a semiconductor interface between different materials, while at the same time retaining graphene $\pi$ conjugation throughout. In this paper we provide a realistic description of QD states and optical excitations for such edge-modulated AGNRs by

(a)

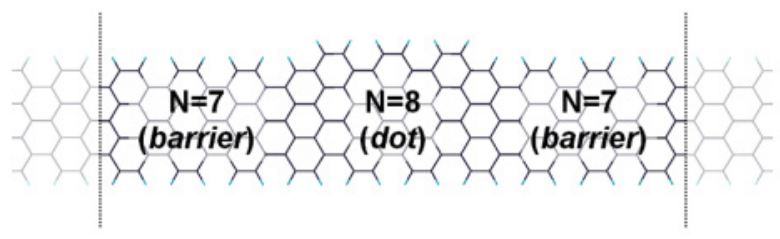

(b)

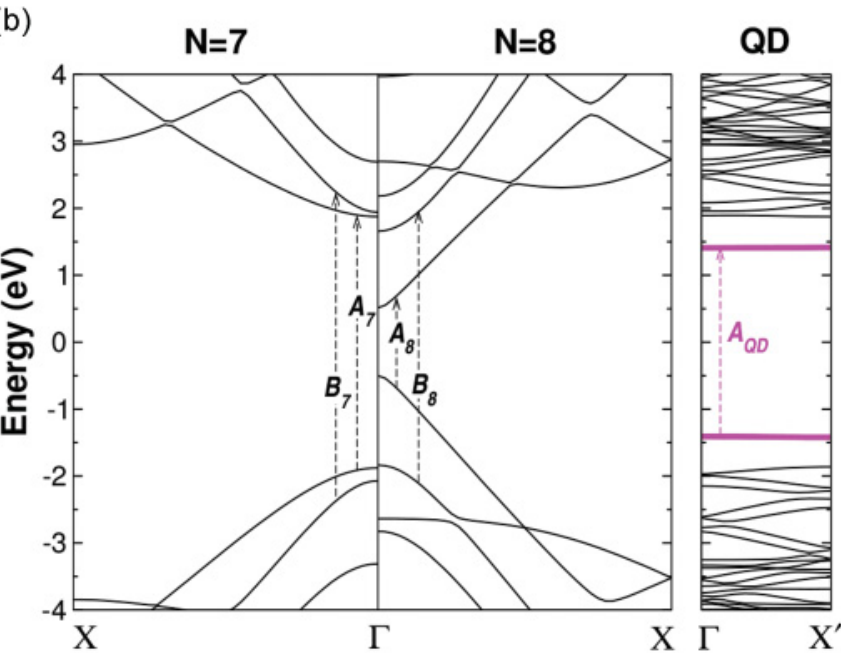

FIG. 1. (Color online) (a) Atomic structure of the modulated graphene nanoribbon. The dot and the barrier regions $(N=8$ and $N=7$, respectively) differ in width by one atomic row. The armchair edges are $\mathrm{H}$ terminated. (b) Quasiparticle band dispersions for the infinite GNRs $N=7$ and $N=8$ (left and middle panels) and for the periodically repeated QD (right panel), whose unit cell is highlighted in (a) by dotted lines. The two nondispersive states (thick magenta lines) are localized in the $\operatorname{dot}(N=8)$ region of the structure. Arrows indicate the lowest optically allowed transitions (see Fig. 2). 
means of state-of-the-art ab initio approaches. Many-body effects, which are known to dominate electronic and optical properties in low-dimensional systems, ${ }^{7,15-17}$ are also included via the $G W$ and Bethe-Salpeter (BS) schemes. ${ }^{18}$ These solidstate techniques, though considerably cumbersome in our case, are crucial to study both dotlike and extended features on equal footing, thus allowing us to obtain quantitative predictions of the optical spectra. All of our results demonstrate that edge-modulated AGNRs offer an original mechanism for the creation of optically active carbon-based QDs with prominent and tunable exciton localization features, which make them suitable for a variety of applications ranging from single-photon emission to optically driven quantum information.

As displayed in Fig. 1, we build our prototypical graphenebased QD by considering a superlattice obtained from the periodically repeated junction of two AGNRs belonging to two different families, $N=7$ (barrier) and $N=8$ (dot), so as to maximize the energy-gap difference and minimize the width variation. Once the two constituents of the superlattice are defined, the length of both the barrier and the dot region will then determine the depth and the number of quantumdot states according to the confinement mechanism described above. Here we choose these parameters in a way to guarantee the presence of a couple of localized states maintaining the feasibility of calculations. In particular, the length of the $N=7$ $(N=8)$ segment is about $3 \mathrm{~nm}(1.3 \mathrm{~nm})$. Note that if the barrier length is too short the first states in the valence and conduction regions are no longer localized. Instead, enlarging the dot region makes the localized states deeper in energy. For comparison, $N=7$ and $N=8$ ideal graphene nanoribbons (GNRs) are also studied. In all systems, dangling bonds at the edges are saturated with monoatomic hydrogen. ${ }^{19}$

The systems described above were fully relaxed by performing density-functional theory (DFT) supercell calculations within the local-density approximation (LDA), as implemented in the QUANTUM ESPRESSO package. ${ }^{20,21}$ In order to improve the band-structure description obtained at the DFTLDA level, we then computed the quasiparticle corrections to the Kohn-Sham eigenvalues within the $G_{0} W_{0}$ approximation for the self-energy operator. In addition, excitonic effects were taken into account by solving the BS equation, which describes the exciton dynamics in terms of the screened quasielectron-quasihole interaction. ${ }^{18}$ From the solution of the BS equation, the absorption spectra were then computed as the imaginary part of the macroscopic dielectric function. The inclusion of the aforementioned many-body effects was done using the YAMBO code. ${ }^{21,22}$

Figure 1(b) depicts the quasiparticle band structures of the studied systems, that is, $N=7, N=8$, and the modulated GNR superlattice. In the superlattice (right panel), the bandgap difference between $N=7$ and $N=8$ acts as a confining potential for the $N=8$ region, giving rise to an effective one-dimensional potential well (periodically repeated). This confining potential is sufficient to localize two states in the well region (see thick nondispersive bands in magenta), which thus behaves as a quantum dot for both electrons and holes. As found for ideal GNRs, ${ }^{7}$ the quasiparticle corrections to the DFT-LDA energy gap are particularly large if compared with standard semiconductors, due to a much weaker screening and

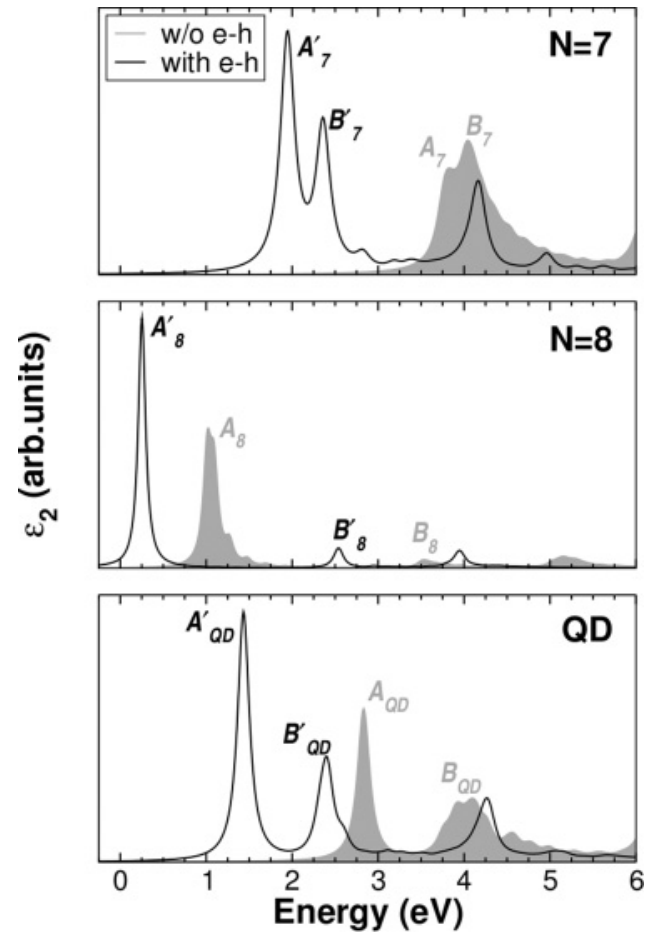

FIG. 2. Optical absorption spectra of $N=7, N=8$, and the modulated GNR (QD). The solid black line represents the spectrum with the inclusion of the electron-hole (e-h) interaction, while the $G W$-RPA (i.e., without the e-h interaction) is in gray. All the spectra are computed for light polarization parallel to the ribbon axis, introducing a Lorentzian broadening of $0.08 \mathrm{eV}$.

the quasi-one-dimensional nature of the systems, which both concur to enhance the electron-electron interaction. This effect is also evident for the superlattice structure, where the LDA energy gap between the localized states is increased from 1.0 to $2.8 \mathrm{eV}$, that is, the $G_{0} W_{0}$ energy gap is almost three times the LDA one. In addition, both ideal and modulated GNRs show an overall stretching of the band structure of about $20 \%$.

The optical absorption spectra for the superlattice and the two subsystems are reported in Fig. 2, as obtained both within the random-phase approximation (RPA, gray shaded area) and including the electron-hole interaction (black line). The main transitions giving rise to the low-energy peaks are indicated by vertical arrows in Fig. 1. As can be seen, the inclusion of excitonic effects, which dramatically modifies both the peak position and the absorption line shape, is crucial to give both qualitative and quantitative predictions of all the optical spectra. The prominent $1 \mathrm{D}$ van Hove singularities characterizing the $G W$-RPA spectra disappear, giving rise to individual excitonic states below the onset of the continuum, with an exceedingly large binding energy. This produces an overall redshift of the spectrum, as opposed to the $G W$ gap opening, together with a change in the relative position of the peaks and possibly in their relative intensity.

In ideal ribbons, the position of the first peak exhibits an oscillating behavior, according to the family classification described above. ${ }^{7}$ This allows one to span an energy window of more than $1.5 \mathrm{eV}$ just by changing the width of one atomic row. In the case of the superlattice, a peak arises between $A_{7}$ 


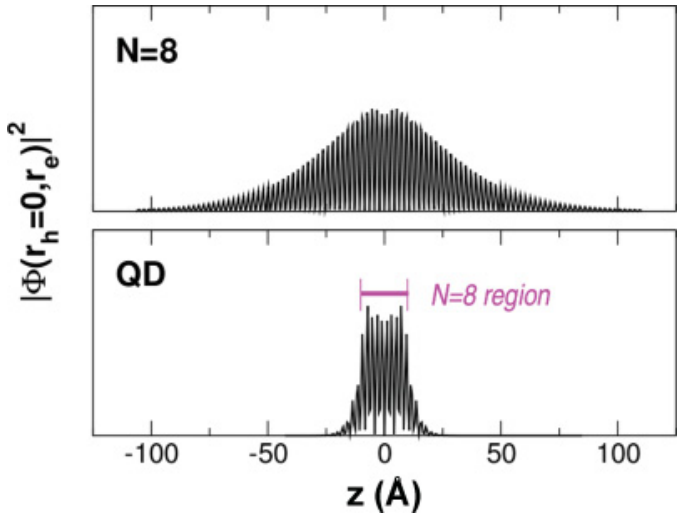

FIG. 3. (Color online) Electron distribution of the lowest excitons for $N=8$ and the edge-modulated GNR (QD), for a fixed hole position (here set as $r_{h}=0$ ). The distribution is plotted along the ribbon axis $z$ after integrating out the other coordinates. For the superlattice $r_{h}$ is chosen to be at the center of the dot $(N=8)$ region.

and $A_{8}$, which corresponds to the interband transition between the states localized in the dot region within the $G W$-RPA picture $\left[A_{\mathrm{QD}}\right.$; see Fig. 1(b), right panel]. As previously mentioned, the structural parameters of the superlattice crucially determine the number and energy location of the confined states, that is, the relative peak position with respect to $A_{7}$ and $A_{8}$. This would further improve the flexibility of this class of system, where a fine tuning of the QD spectrum can be enabled both by interfacing different GNRs and by changing the dot and/or barrier length within a given superstructure.

Let us now focus in more detail on the nature of the first peak of the edge-modulated GNR. When excitonic effects are included, the first optically active excitation still has a predominantly localized nature (i.e., $78 \%$ of the weight is given by the transition between localized states confined in the $N=8$ region). Thus the markedly different correction to the $G W$-RPA spectra as compared to the ideal $N=8$ GNR has to be ascribed to the additional confining potential introduced by the edge modulation, the first one being almost twice as large. This change in the confinement properties is also apparent in the excitonic wave function plotted along the ribbon axis (Fig. 3): In the presence of the $N=7$ barrier, the overall shape of the exciton envelope function changes from Gaussian (top panel) to steplike (bottom panel), with a significant reduction of the spatial extension and an exponential decay in the barrier region. Nonetheless, the exciton preserves a Wannier-like character [Fig. 4(a)], as found for ideal AGNRs. ${ }^{7}$

As described above, while the first excitation has mainly a dotlike character, it also contains non-negligible contributions coming from higher-energy levels, thus acquiring a mixed character. This is also true for higher excitations, which arise from combinations of single-particle states with different localization properties. For instance, the second peak is made up of several excitonic states, almost degenerate in energy, each of them combining contributions from single-particle states localized in the dot, in the barrier, or from resonances delocalized over the whole system. The mixed character of the excitations, as well as the presence of both dotlike and bulklike (a)

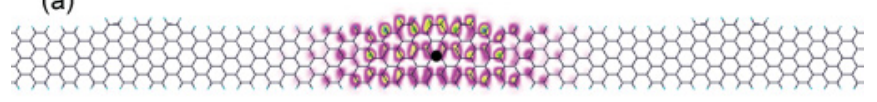

(b)

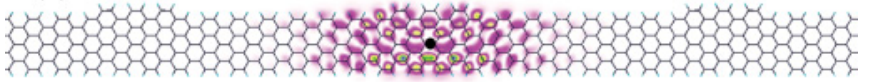

(c)

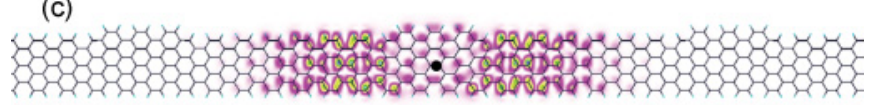

(d)

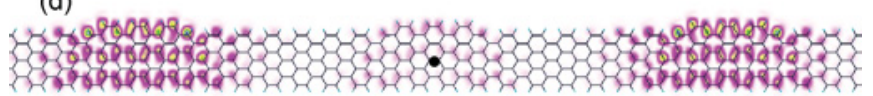

FIG. 4. (Color online) In-plane electron distribution of the (a) first bright and (b)-(d) few higher dark excitons for the QD superlattice, where the black dot indicates the fixed hole.

excitons, must be ascribed to the unique nature of the system: A straddling junction between regions with different energy gaps is realized here using the same material, thus preserving $\pi$ conjugation at the junction interface. ${ }^{23}$ This is indeed different from what happens in common heterojunctions made of different materials. ${ }^{24}$

In order to gain further insight into the optical activity of such systems, we have investigated the presence of dark states in the low-energy window for the periodically repeated QD structure. Figure 4 illustrates the in-plane electron distribution of the the first (bright) exciton [Fig. 4(a)] and a few higher optically inactive excitons [Figs. 4(b)-4(d)] of the QD, plotted at a fixed hole position (black dot). The edge modulations do not introduce optically inactive states below the first absorption peak, inheriting the behavior of ideal ribbons. ${ }^{7}$ Several dark states of distinct nature appear instead in the energy region between the first and second peaks [Figs. 4(b)4(d)]. In addition to the more common dipole forbidden states [see, e.g., Fig. 4(b)], we find excitons that couple singleparticle states spatially localized in different regions of the superstructure. Optical inactivity thus results from the small overlap of the electron and hole wave functions [see, e.g., Fig. 4(c), where the hole is in the dot, while the electron is localized in the barrier]. A third type of exciton is shown in Fig. 4(d), where the hole is in the dot region and the electron is localized in its nearest replicas.

To summarize, we have investigated the electronic and optical properties of edge-modulated AGNRs including quasiparticle corrections and excitonic effects. Exploiting the oscillating behavior of their energy gap, we have demonstrated that the simple addition (or removal) of single dimeric lines along the ribbon width is sufficient to obtain contextual confinement of both electrons and holes. We show that these nanostructures can act as optically active QDs, whose properties are significantly modified by many-body effects. The coexistence of dotlike and extended excitations, as well as the fine tunability of optical spectra, is a unique feature that make these systems conceptually different from conventional QDs. These results offer a tantalizing perspective, especially in light of the recent production of atomically controlled armchair-edged GNRs. 
The authors thank F. Troiani and G. Goldoni for fruitful discussions. This work was partially funded by "Fondazione Cassa di Risparmio di Modena," Italian Ministry of Foreign
Affairs, and MIUR-FIRB Grant ItalNanoNet. CPU time was granted by CINECA through INFM-CNR.
*Author to whom correspondence should be addressed: deborah.prezzi@unimore.it

†Present address: Dipartimento di Fisica, Università di Roma "La Sapienza," I-00185 Roma, Italy.

${ }^{1}$ A. H. Castro Neto et al., Rev. Mod. Phys. 81, 109 (2009); A. Geim, Science 324, 1530 (2009).

${ }^{2}$ M. Y. Han, B. Ozyilmaz, Y. Zhang, and P. Kim, Phys. Rev. Lett. 98, 206805 (2007); Z. Chen et al., Physica E 40, 228 (2007).

${ }^{3}$ K. Nakada, M. Fujita, G. Dresselhaus, and M. S. Dresselhaus, Phys. Rev. B 54, 17954 (1996); Y.-W. Son, M. L. Cohen, and S. G. Louie, Phys. Rev. Lett. 97, 216803 (2006).

${ }^{4}$ D. Wei and Y. Liu, Adv. Mater. 22, 3225 (2010); S. Roche, Nature Nanotech. 6, 8 (2010).

${ }^{5}$ J. Cai et al., Nature (London) 466, 470 (2010).

${ }^{6}$ D. Prezzi, D. Varsano, A. Ruini, A. Marini, and E. Molinari, Phys. Status Solidi B 244, 4124 (2007).

${ }^{7}$ D. Prezzi, D. Varsano, A. Ruini, A. Marini, and E. Molinari, Phys. Rev. B 77, 041404 (2008).

${ }^{8}$ L. Yang, M. Cohen, and S. Louie, Nano Lett. 7, 3112 (2007).

${ }^{9}$ C. Cocchi, D. Prezzi, A. Ruini, M. J. Caldas, and E. Molinari, J. Phys. Chem. Lett. 2, 1315 (2011).

${ }^{10}$ C. Stampfer, J. Guttinger, S. Hellmuller, F. Molitor, K. Ensslin, and T. Ihn, Phys. Rev. Lett. 102, 056403 (2009); P. Gallagher, K. Todd, and D. Goldhaber-Gordon, Phys. Rev. B 81, 115409 (2010); M. Y. Han, J. C. Brant, and P. Kim, Phys. Rev. Lett. 104, 056801 (2010).

${ }^{11}$ T. Shimizu et al., Nature Nanotech. 6, 45 (2011).

${ }^{12}$ P. G. Silvestrov and K. B. Efetov, Phys. Rev. Lett. 98, 016802 (2007); Z. F. Wang et al., Appl. Phys. Lett. 91, 053109 (2007);
H. Sevinçli, M. Topsakal, and S. Ciraci, Phys. Rev. B 78, 245402 (2008); B. Borca et al., Phys. Rev. Lett. 105, 036804 (2010); A. K. Singh et al., ACS Nano 4, 3510 (2010).

${ }^{13}$ L. Ponomarenko et al., Science 320, 356 (2008).

${ }^{14}$ Semiconductor Macroatoms, edited by F. Rossi (Imperial College Press, London, 2005).

${ }^{15}$ D. Prezzi and E. Molinari, Phys. Status Solidi A 203, 3602 (2006).

${ }^{16}$ A. Ruini, M. J. Caldas, G. Bussi, and E. Molinari, Phys. Rev. Lett. 88, 206403 (2002).

${ }^{17}$ E. Chang, G. Bussi, A. Ruini, and E. Molinari, Phys. Rev. Lett. 92, 196401 (2004); J. Maultzsch, R. Pomraenke, S. Reich, E. Chang, D. Prezzi, A. Ruini, E. Molinari, M. S. Strano, C. Thomsen, and C. Lienau, Phys. Rev. B 72, 241402 (2005).

${ }^{18}$ For a recent review on $G W$-BSE see, e.g., G. Onida, L. Reining, and A. Rubio, Rev. Mod. Phys. 74, 601 (2002).

${ }^{19}$ T. Wassmann, A. P. Seitsonen, A. M. Saitta, M. Lazzeri, and F. Mauri, Phys. Rev. Lett. 101, 096402 (2008).

${ }^{20}$ P. Giannozzi et al., J. Phys. Condens. Matter 21, 395502 (2009).

${ }^{21}$ See Supplementary Material at http://link.aps.org/supplemental/ 10.1103/PhysRevB.84.041401 for details of the calculation.

${ }^{22}$ A. Marini, C. Hogan, M. Grüning, and D. Varsano, Comput. Phys. Commun. 180, 1392 (2009).

${ }^{23}$ Type-II nanojuctions with peculiar optical properties can also be obtained from AGNRs by means of edge functionalization with appropriate chemical groups. ${ }^{9,25}$

${ }^{24}$ See e. g. BN-C nanoribbon heterostructures by Sevinçli et al. [12].

${ }^{25}$ C. Cocchi, A. Ruini, D. Prezzi, M. J. Caldas, and E. Molinari, J. Phys. Chem. C 115, 2969 (2011). 